\title{
Tensile Stress-Strain Modeling of Pseudostrain Hardening Cementitious Composites
}

\author{
By Tetsushi Kanda, ${ }^{1}$ Zhong Lin, ${ }^{2}$ and Victor $\mathrm{C} . \mathrm{Li}^{3}$
}

\begin{abstract}
This study proposes a new theoretical approach for predicting the tensile stress-strain relation of random short-fiber-reinforced cement composites showing pseudostrain hardening. This approach is grounded on the solid basis of micromechanics, which describes the pseudostrain hardening phenomenon in terms of constitutive properties of the fiber, matrix, and fiber/matrix interface. The proposed modeling requires theoretical treatment of an inelastic strain due to multiple cracking. This modeling is achieved by employing a probabilistic description of initial flaw size distribution, which should be known for predicting the stress-strain relation. This study proposes a practical method for this identification using the tensile test result of a reference composite. A comparison with the test data indicates that the proposed model is capable of reasonably reproducing the stressstrain relation of "similar" composites. Such composites have a configuration similar to the reference configuration but different in fiber volume fraction and fiber length. Finally, the proposed theory is a potentially powerful tool for tailoring composites to satisfy targeted structural performance.
\end{abstract}

\section{INTRODUCTION}

Pseudostrain hardening (PSH) cement composites reinforced with short random fibers have been extensively investigated in recent years [e.g., Leung (1996) and Li (1993)]. These random short-fiber-reinforced cement composites (RSFRCC) with PSH are defined as composites that exhibit multiple cracking perpendicular to the loading axis under uniaxial tensile loading. The multiple cracking is accompanied by one to two orders of higher tensile strain capacity and fracture toughness compared with conventional RSFRCCs. It is expected that the structural performance of elements (e.g., seismic resistant elements) with PSH-RSFRCCs can be greatly enhanced (Naaman and Reinhardt 1995). Detailed investigations have been conducted to design these PSH-RSFRCCs, adopting micromechanics by $\mathrm{Li}$ and coworkers [e.g., $\mathrm{Li}$ and Leung (1992)]. As a result, the micromechanics-based design guidelines have been established for PSH-RSFRCCs.

However, the micromechanics-based prediction of PSHRSFRCC's tensile stress-strain relation has been less clarified, which is an important characteristic for structural design. A major obstacle in tensile stress-strain modeling is the characterization of an inelastic strain due to matrix cracking. This inelastic strain was originally investigated for continuous aligned fiber-reinforced composites by Aveston et al. (1971), in which matrix cracking stress was simply assumed uniform in each of the multiple cracks. Their results were then extended for composites reinforced with randomly distributed long fibers (Aveston and Kelly 1973). Following these research works, matrix cracking of composites under tension has been extensively investigated in the field of ceramics. Stress at cracking was derived as a function of micromechanical parameters representing the initial flaw size and the fiber's crack bridging performance (Marshall et al. 1985; Marshall and Cox 1987). Furthermore, cracking was statistically examined, and its stochastic aspects were analytically clarified (Beyerle et al. 1992; Cho et al. 1992; Zok and Spearing 1992; Spearing and

${ }^{1}$ Sr. Res. Engr., Kajima Tech. Res. Inst., 2-19-1 Tobitakyu, Chofu-shi, Tokyo, 182-0036, Japan.

${ }^{2}$ Sr. Engr., Delphi Automotive Systems, 5725 Delphi Dr., Troy, MI 48098.

${ }^{3}$ Prof. and Dir., Advanced Civ. Engrg. Mat. Res. Lab., Dept. of Civ. and Envir. Engrg., Univ. of Michigan, Ann Arbor, MI 48109-2125.

Note. Associate Editor: Nemkumar Banthia. Discussion open until October 1, 2000. To extend the closing date one month, a written request must be filed with the ASCE Manager of Journals. The manuscript for this paper was submitted for review and possible publication on February 3, 1999. This paper is part of the Journal of Materials in Civil Engineering, Vol. 12, No. 2, May, 2000. C)ASCE, ISSN 0899-1561/00/00020147-0156/\$8.00 + \$.50 per page. Paper No. 20168.
Zok 1993). Based on these results, inelastic strain due to matrix cracking was modeled in relation with crack evolution [e.g., He et al. (1994)]. An analytic model for the stress-strain relation was then proposed for ceramic composites, which are restricted on the condition that fibers are aligned and continuous (Evans et al. 1994). However, few attempts have yet been made to extend these theories for random short-fiber-reinforced composites.

For RSFRCCs, tensile stress-strain models have recently been suggested using micromechanical properties of constitutive materials while essentially requiring rather complicated numerical calculation. For example, the theory by Aveston and Kelly (1973) has been extended such that the realistic behavior of short fibers in composites (e.g., debonding, pullout, and rupture) was accounted for (Kullaa 1998). Statistical aspects in matrix cracking were not considered in this theory. Moreover, a finite-element method (FEM) simulation scheme was proposed accounting for the pullout behavior of a single fiber, in which matrix cracking is treated as a random variable (Alwan 1994). These models were reported to yield stress-strain curves of RSFRCCs consistent with the test results.

For PSH-RSFRCC structural elements, an advanced FEM analysis approach has recently been proposed (Kabele and Horii 1996). This FEM model implements a constitutive law that expresses multiaxis macroscopic behavior of PSHRSFRCCs. This constitutive law requires that the uniaxial tensile stress-strain relation be given. Therefore, expressing the uniaxial stress-strain relation in terms of the micromechanical parameters is a basis for assessing the more complicated structural behavior of PSH-RSFRCC elements, in which PSHRSFRCCs are usually in multiaxial stress states.

The current study proposes a new tensile stress-strain model for PSH-RSFRCCs, with emphasis on maintaining mathematical simplicity while grounded on the solid basis of micromechanics. The micromechanics employed has been established to describe the PSH phenomenon in terms of constitutive properties of the fiber, matrix, and fiber/matrix interface (Li and Leung 1992). This fundamental micromechanics has provided reasonable explanations for the experimental observations of multiple cracking [e.g., Li et al. (1995)]. The current theory represents an extension of the approach adopted by $\mathrm{Wu}$ and $\mathrm{Li}$ (1995b), in which the multiple cracking sequence was treated statistically, and the flaw size distribution was computer simulated as a Monte Carlo process ( $\mathrm{Wu}$ and Li 1995b). As a result, the present model provides simple analytic formulas for the stress-strain relation of PSH-RSFRCCs.

In the present study, the following three major issues, which relevant to the modeling of the tensile stress-strain relation, are addessed: (1) A simple geometric representation of the 
stress-strain relation; (2) a constitutive model for predicting ultimate crack spacing; and (3) a procedure for identifying flaw size distribution. The resulting stress-strain theory is then substantiated with the experimental test results. Finally, the implications of this theory are discussed in relation to the design of composites as structural materials.

\section{THEORETICAL TREATMENT OF STRESS-STRAIN RELATION}

\section{Tensile Stress-Strain Representation}

One of the most distinct features of PSH-RSFRCCs is that their inelastic tensile behavior can be represented by stressstrain relations. This is different from conventional RSFRCCs, whose tensile behavior proceeds to the postpeak softening stage immediately after first cracking. The softening stage must be represented by a stress-crack opening displacement (COD) relation as illustrated in Fig. 1. For the conventional RSFRCCs, this tension softening behavior should be taken into account in structural design. On the other hand, the prepeak stress-strain behavior is important for predicting structural response as well as postpeak behavior when PSHRSFRCC is used. For the PSH-RSFRCCs, tensile stress is maintained after first cracking, accompanying significant strain extension as depicted in Fig. 2. This figure illustrates that tensile behavior can apparently be expressed with a stress-strain relation up to the ultimate state, when damage localization initiates. (Note that the stress-COD relation should be used after the ultimate state.)

\section{Outline of Proposed Theory}

The tensile stress-strain relation for PSH-RSFRCCs is characterized by two states, the first crack state and the ultimate

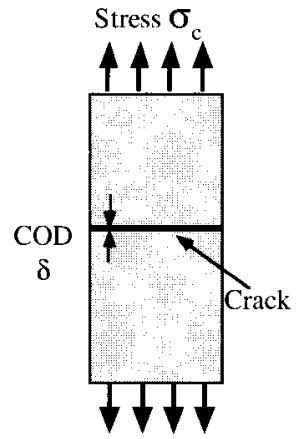

(a) Crack generation in conventional composite

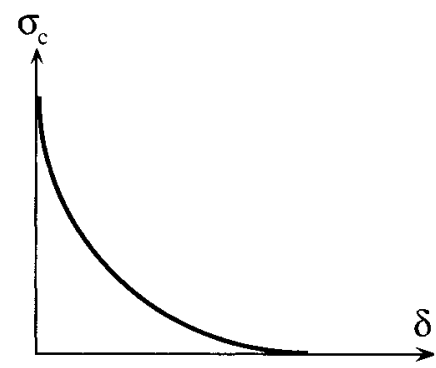

(b) Stress-COD relation
FIG. 1. Tensile Behavior Representation for Conventional RSFRCC

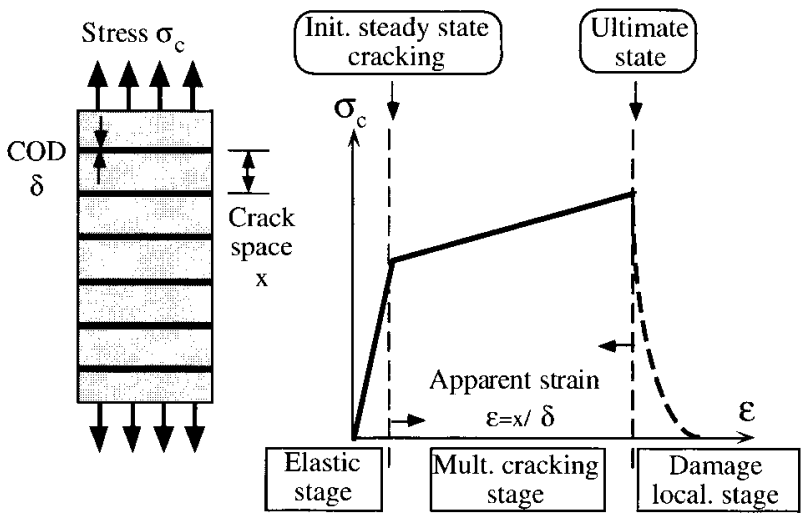

(a) Multiple crack evolution in PSH composite

(b) Stress-Strain relation

FIG. 2. Tensile Behavior Representation for PSH-RSFRCC

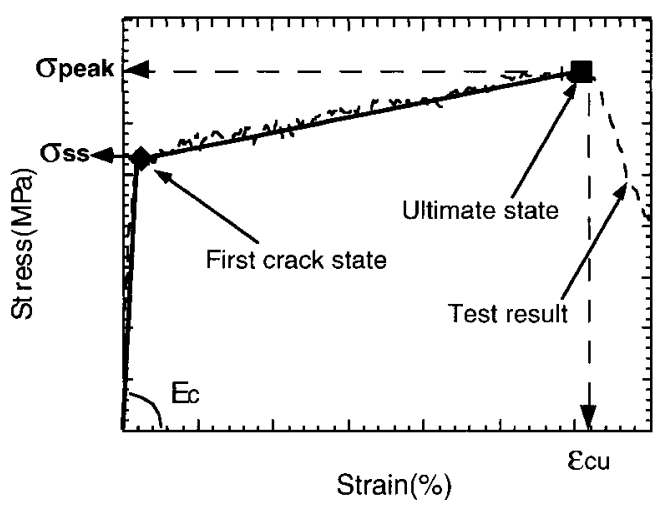

FIG. 3. Tensile Stress-Strain Representation; $-=$ Model; $---=$ Experimental Data

state. These two states are illustrated in Fig. 3, which shows an actual tensile stress-strain curve of PSH-RSFRCCs and its geometric bilinear representation. The first crack state refers to the first bend-over point and is assumed to be equal to the steady-state cracking stress $\sigma_{s s}$ (further defined below), when multiple cracking initiates. The ultimate state refers to the peak tensile stress state when multiple cracking terminates. The bilinear representation of $\sigma(\varepsilon)$ in (1) requires the theoretical prediction of ultimate strain $\varepsilon_{c u}$, the steady-state cracking stress $\sigma_{s s}$, ultimate stress $\sigma_{\text {peak }}$, and composite elastic modulus $E_{c}$ (Fig. 3)

$$
\sigma(\varepsilon)=\left\{\begin{array}{l}
E_{c} \varepsilon, \quad \varepsilon \leq \sigma_{s s} / E_{c} \\
\sigma_{i}+E_{i e} \varepsilon, \quad \varepsilon>\sigma_{s s} / E_{c}
\end{array}\right.
$$

where

$$
E_{i e}=\left(\frac{\sigma_{\text {peak }}-\sigma_{s s}}{\varepsilon_{c u}-\sigma_{s s} / E_{c}}\right) ; \quad \sigma_{i}=\sigma_{s s}\left(1-\frac{E_{i e}}{E_{c}}\right)
$$

Supposed $E_{c}$ is known, and three major parameters, $\varepsilon_{c u}, \sigma_{s s}$, and $\sigma_{\text {peak }}$, need to be theoretically identified to complete (1). The procedure for identifying these major unknown parameters is outlined in the first step (Step I) in Fig. 4. In the first step, estimating $\sigma_{s s}$ and $\sigma_{\text {peak }}$ is completed, based on published theory, which relates these parameters to fiber, matrix, and interface properties ( $\mathrm{Li}$ and Leung 1992). However, $\varepsilon_{c u}$ has not been comprehensively modeled in past studies, and its identification is not completed in the first step. This identification is the focus of the present study and needs three additional steps (Steps II-IV) in Fig. 4, which are described in detail in subsequent sections. Identifying $\sigma_{s s}$ and $\sigma_{\text {peak }}$ in first step of Fig. 4 is explained in more detail below.

Steady-state cracking stress is estimated from composite crack bridging stress $\sigma_{c}$, which represents the apparent closing pressue due to fiber bridging acting on the composite crack plane, and cracking stress level $\sigma_{f c}$. Moreover, $\sigma_{f c}$ is defined as the stress level at which each of the multiple cracks propagates, when each crack at a different part of the specimen has a different size. The stresses $\sigma_{c}$ and $\sigma_{f c}$ were derived based on fracture mechanics and micromechanics as follows ( $\mathrm{Li}$ and Leung 1992):

$$
\begin{gathered}
\frac{\hat{\sigma}_{c}}{g}=\sqrt{2 \bar{c}}-\frac{\bar{c}}{2} \\
\frac{\hat{\sigma}_{f c}}{g}=\left(\frac{2 \sqrt{2}}{3} \sqrt{\bar{c}}-\frac{\bar{c}}{4}\right)+\frac{\sqrt{\pi}}{2 \bar{c}} \bar{K}
\end{gathered}
$$

where

$$
\hat{\sigma}_{f c}=\frac{\sigma_{f c}}{\sigma_{0}} ; \quad \hat{\sigma}_{c}=\frac{\sigma_{c}}{\sigma_{0}} ; \quad \sigma_{0}=\frac{V_{f} \tau_{i}}{2}\left(\frac{L_{f}}{d_{f}}\right) ; \quad g=\frac{2}{4+f^{2}}\left(1+e^{\pi f / 2}\right)
$$




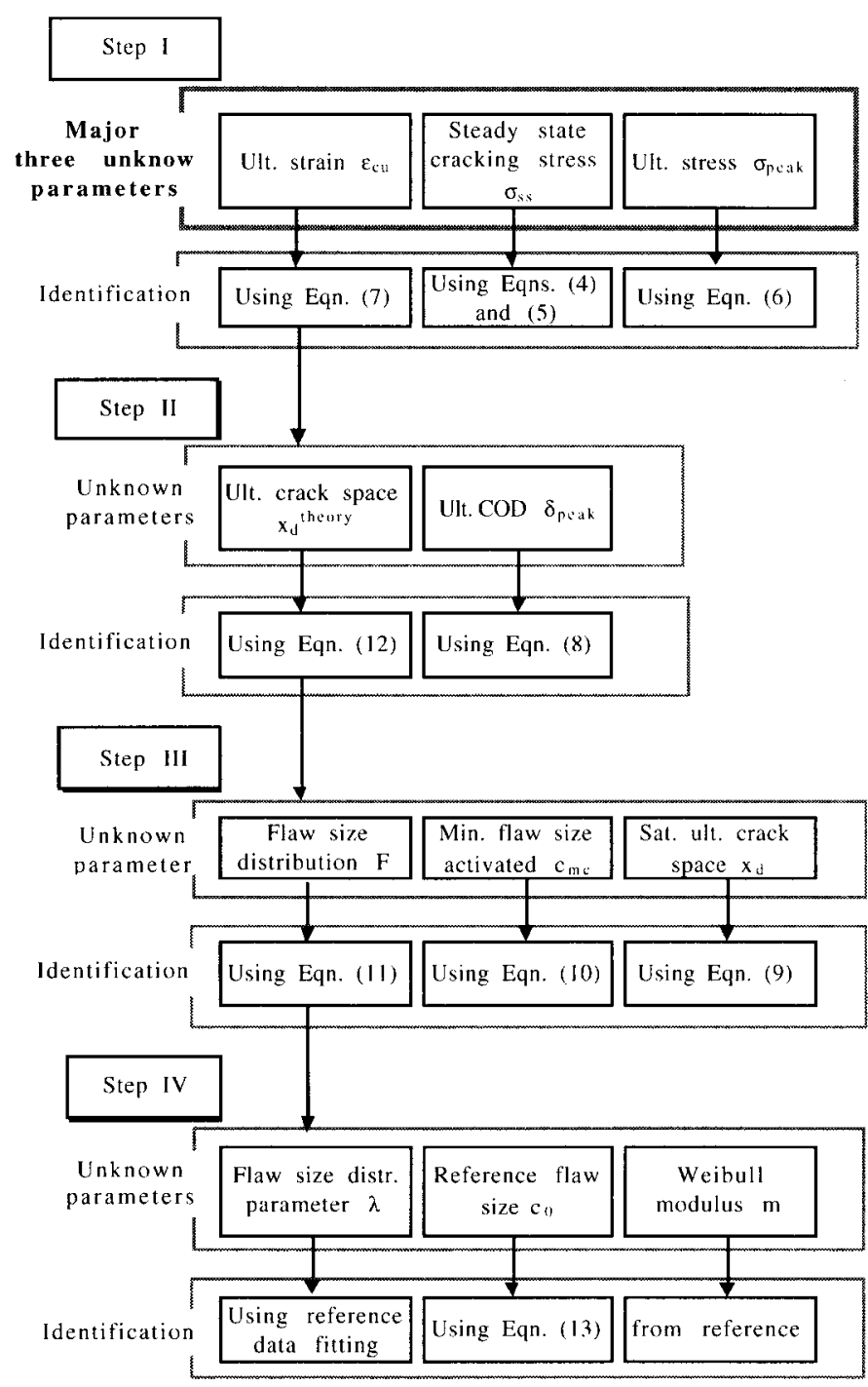

FIG. 4. Outline of Proposed Stress-Strain Theory

$f=$ snubbing coefficient; $\tau_{i}=$ frictional bond strength; $V_{f}=$ fiber volume fraction; $L_{f}=$ fiber length; $d_{f}=$ fiber diameter

$$
\begin{gathered}
\bar{c}=\frac{\sqrt{\hat{c}}}{\hat{\delta}^{*}} ; \quad \hat{c}=\frac{c}{c_{i}} ; \quad \hat{\delta}^{*}=\frac{2 \tau}{E_{f}(1+\eta)} \frac{L_{f}}{d_{f}} ; \quad \eta=\frac{V_{f} E_{f}}{V_{m} E_{m}} ; \\
\bar{K}=\frac{K_{\mathrm{tip}}}{\sigma_{0} \sqrt{c_{i}}} \frac{1}{g \hat{\delta}^{*}} ; \quad c_{i}=\left(\frac{L_{f} E_{c}}{2 K_{\mathrm{tip}}}\right)^{2} \frac{\pi}{16\left(1-v^{2}\right)^{2}}
\end{gathered}
$$

$c=$ flaw radius $; E_{f}=$ fiber elastic modulus; $V_{m}=$ matrix volume fraction of matrix, $E_{m}=$ elastic modulus of matrix; $K_{\text {tip }}=$ crack tip fracture toughness; $E_{c}=$ elastic modulus of composite; and $\nu=$ Poisson's ratio of composite. In (2) and (3), $\sigma_{c}$ and $\sigma_{f c}$ are expressed as a function of flaw size $c$, and they are schematically shown in Fig. 5(a). Eqs. (2) and (3) were essentially developed for "flexible" fibers such as polymer fibers that have no bending stiffness. Furthermore, neither fiber rupture in crack bridging action nor chemical bonding between fiber/ matrix interface was taken into account. Because it is based on (2) and (3), the stress-strain theory in this paper has the same limitation in the properties of fiber and fiber interfaces.

Steady-state cracking occurs when $\sigma_{c}$ becomes equal to $\sigma_{f c}$ in Fig. 5(a). Hence, steady-state cracking stress $\sigma_{s s}$ can be derived as (4), using (2) and (3) (Li and Leung 1992)

$$
\hat{\sigma}_{s s}=g\left(\sqrt{2 \bar{c}_{s}}-\frac{\bar{c}_{s}}{2}\right)
$$

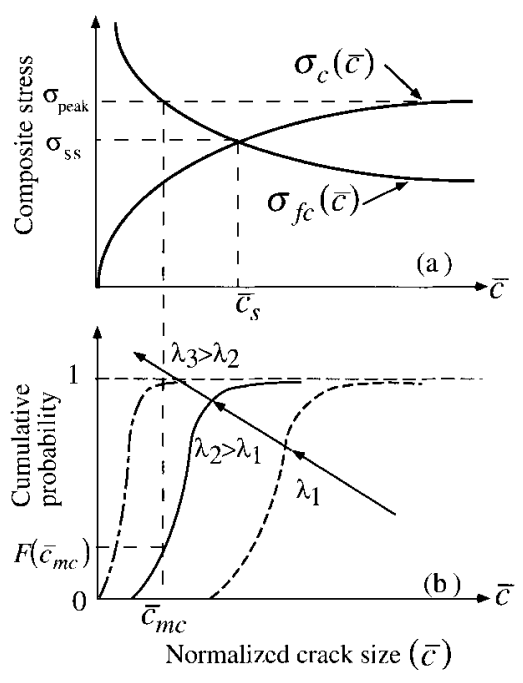

FIG. 5. (a) Composite Cracking Stress and Midcrack Bridging Stress; (b) Cumulative Probability, as Function of Crack Size

where

$$
\hat{\sigma}_{s s}=\frac{\sigma_{s s}}{\sigma_{0}} ; \quad \bar{c}_{s}=\frac{\sqrt{\hat{c}_{s}}}{\hat{\delta}^{*}} ; \quad \hat{c}_{s}=\frac{c_{s}}{c_{i}}
$$

The term $\bar{c}_{s}$ in (4) is the flaw size as steady-state cracking occurs and is determined by solving

$$
\frac{2}{\sqrt{\pi}} \bar{c}_{s}\left(\frac{\sqrt{2 \bar{c}_{s}}}{3}-\frac{\bar{c}_{s}}{4}\right)=\bar{K}
$$

The second unknown, peak bridging stress $\sigma_{\text {peak }}$, can be evaluated as the maximum value in (2) ( $\mathrm{Li}$ and Leung 1992)

$$
\sigma_{\text {peak }}=g \sigma_{0}
$$

Hence, the remaining task is to theoretically express $\varepsilon_{c u}$ in terms of micromechanical parameters. As an intermediate step, $\varepsilon_{c u}$ can be written as follows (Lin and Li 1997):

$$
\varepsilon_{c u}=\frac{\delta_{\text {peak }}}{x_{d}^{\text {theory }}}
$$

where $\delta_{\text {peak }}=$ ultimate COD; and $x_{d}^{\text {theory }}=$ theoretically predicted ultimate crack spacing. The assumption in establishing (7) is illustrated in Fig. 6. Fig. 6(a) shows the stress-strain relation of PSH-RSFRCC in uniaxial tension loading, in which the ultimate state is specified with $\sigma_{\text {peak }}$ and $\varepsilon_{c u}$. At this ultimate state, a composite generates cracks as shown in Fig. 6(b). All of these cracks are assumed to have $\delta_{\text {peak }}$ (i.e., COD corresponding to bridging stress $\left.\sigma_{\text {peak }}\right)$ as shown in Fig. 6(c). The crack spacing for PSH-RSFRCCs decreases with increasing applied load and with accumulation of multiple cracking and is assumed to reach ultimate crack spacing $x_{d}^{\text {test }}$ at the ultimate state. Finally $x_{d}^{\text {theory }}$ is defined as the theoretical prediction of $x_{d}^{\text {test }}$, and $\varepsilon_{c u}$ is then expressed with (7). Eq. (7) assumes that both crack opening and spacing are uniform over the specimen gauge length at the ultimate state.

Therefore, the focus in this study is reduced to identifying the two parameters on the right-hand side of (7) after Step I in Fig. 4.

\section{Theoretical Prediction of Ultimate Strain}

Identification of two parameters, $\delta_{\text {peak }}$ and $x_{d}^{\text {theory }}$, in Step II of Fig. 4 is explained in this section. For the first parameter $\delta_{\text {peak }}$, Lin and Li (1997a) found that neglecting the slip-hardening phenomenon in single fiber behavior results in significantly underestimating $\delta_{\text {peak }}$ in RSFRCC's stress-COD relation. 


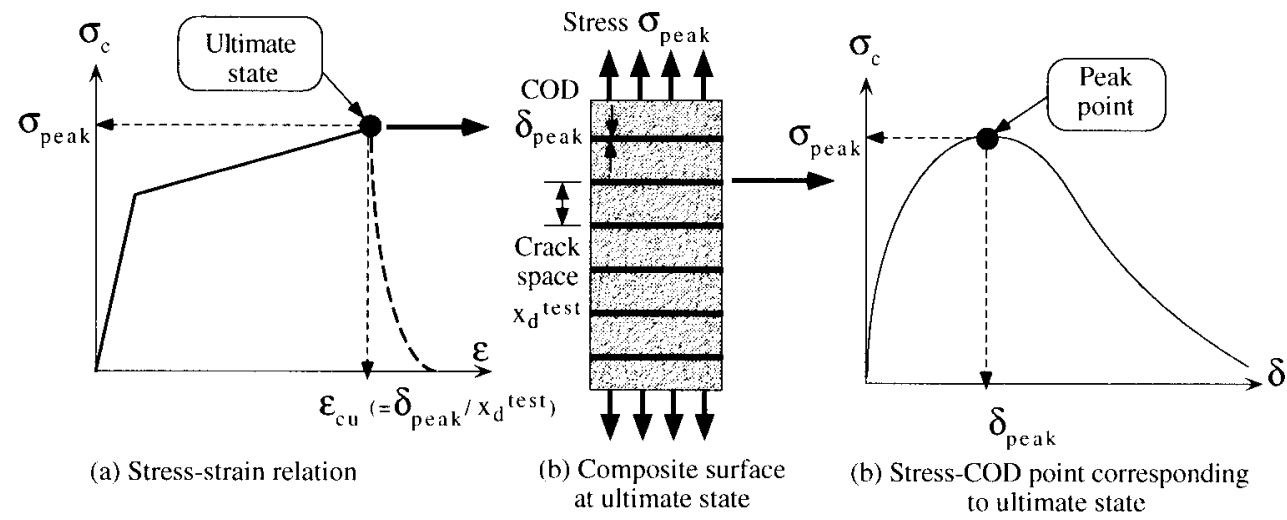

FIG. 6. Assumption in Ultimate Strain Expression

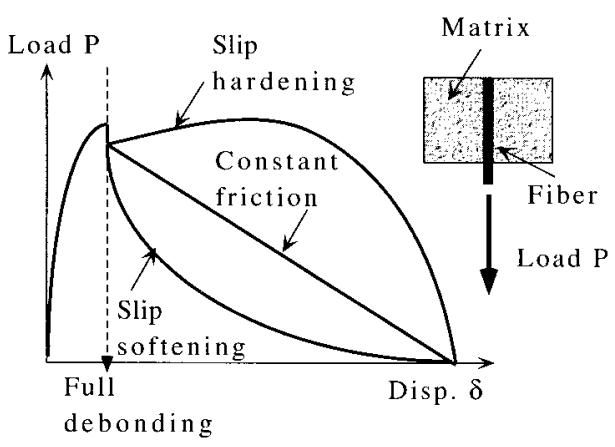

FIG. 7. Three Typical Profiles of Single Fiber Pullout Curve

The slip-hardening phenomenon is often observed in bond behavior between polymeric fibers and cement matrix ( $\mathrm{Li}$ and Stang 1996). Fig. 7 illustrates three typical load-displacement curves reported in single fiber pullout tests. Slip-hardening is originated by increasing the frictional resistance with slip distance after full debonding. This friction increase is considered due to the abrasion on the soft polymeric fiber surface against cement hydration particles ( $\mathrm{Li}$ and Stang 1996). When the fiber surface is hard as in the case of a steel fiber, the tensionsoftening behavior is observed as shown in Fig. 7 (Naaman and Najm 1991).

In RSFRCC's stress-COD relation, $\delta_{\text {peak }}$ has recently been predicted considering the slip-hardening phenomenon in the behavior of a single polymeric fiber pulled out of a cementitious matrix (Lin and $\mathrm{Li}$ 1997)

$$
\hat{\delta}_{\text {peak }}=\frac{b-\sqrt{b^{2}-4 a}}{2}
$$

where

$$
b=\frac{2 a_{2}-3 a_{1}}{4 a_{2}} ; \quad a=\frac{a_{1}-2}{4 a_{2}} ; \quad a_{1}=\frac{\beta_{1} L_{f}}{2 d_{f}} ; \quad a_{2}=\frac{\beta_{2} L_{f}^{2}}{4 d_{f}^{2}}
$$

$\beta_{1}=$ first-order nondimensional hardening parameter; and $\beta_{2}$ $=$ second-order nondimensional hardening parameter. The parameters $\beta_{1}$ and $\beta_{2}$ are phenomenological (interface) parameters determined from the load-displacement relation obtained in the pullout test of a single fiber embedded in the matrix.

The second parameter $x_{d}^{\text {theory }}$ is estimated via a newly proposed theory. The specific case of PSH-RSFRCCs involving full saturation of multiple cracking has been previously studied (Kanda and $\mathrm{Li}$ 1998). The ultimate crack spacing with full crack saturation $x_{d}$ (saturated ultimate crack spacing, hereafter) depends on transferred stress from bridging fiber at crack plane to noncracked matrix. This stress transfer is achieved via interfacial shear stress between the fiber and matrix. The saturated ultimate crack spacing $x_{d}$ can be estimated as the mini- mum distance necessary for overcoming matrix cracking stress by transferred stress. This saturated ultimate crack spacing is expressed as (Wu and $\mathrm{Li}$ 1995a)

$$
x_{d}=\frac{L_{f}-\sqrt{L_{f}^{2}-2 \pi \psi L_{f} x}}{2}
$$

where

$$
\psi=\frac{4}{\pi g} ; \quad x=\frac{V_{m} \sigma_{m u} d_{f}}{4 V_{f} \tau_{i}}
$$

where $\sigma_{m u}=$ tensile strength of matrix.

The saturation of multiple cracking is not a necessary condition for PSH behavior as demonstrated using uniaxial tensile tests by Kanda and Li (1998). In these tests, RSFRCCs showed PSH behavior, but larger ultimate crack spacing than $x_{d}$ was observed in most of those composites (so-called unsaturated PSH-RSFRCCs). This observation implies the deficiency of simply using $x_{d}$ to evaluate the ultimate crack spacing. The existing crack spacing theory (Wu and Li 1995a) should be extended to cover both saturated and unsaturated PSHRSFRCCs. Thus, the scope of this study is further narrowed down to establishing a new model for evaluating unsaturated ultimate crack spacing, as shown in Step III of Fig. 4. The theory is presented in greater detail below.

\section{Theory for Predicting Ultimate Crack Spacing}

The new crack spacing theory, which examines the multiple cracking sequence, is introducted by extending previous research. For saturated PSH-RSFRCCs, the multiple cracking sequence was demonstrated to be successfully reproduced with the Monte Carlo simulation (Wu and Li 1995b). The simulation was conducted under the following hypothesis: (1) The initial flaw size distribution can be represented as a random process that governs crack evolution; and (2) crack evolution is terminated if $\sigma_{f c}>\sigma_{\text {peak }}$. This simulation neglects the micromechanical parameters' variability other than flaw size. The current study employs the same fundamental ideas in the simulation method of Wu and $\mathrm{Li}$ (1995b) and attempts to construct a crack spacing theory for unsaturated PSH-RSFRCCs as well as saturated ones. The resulting analytical theory is simpler than the original computer simulation scheme. It should be noted that the current theory reduces to identical results of the existing one (Wu and $\mathrm{Li}$ 1995a) when cracks are fully saturated.

In the current crack spacing theory, it is assumed that unsaturated crack spacing can be evaluated employing the probability of potentially propagating flaws involved in a tensile specimen. Cracks are not to be generated with spacing less than $x_{d}$, as the matrix stress cannot attain cracking stress level within length $x_{d}$ from a crack plane in theory (Aveston et al. 1971). Furthermore, the ratio $x_{d} / x_{d}^{\text {test }}$, where $x_{d}^{\text {test }}$ represents ob- 
served crack spacing in the tests, may be expected to be related to flaw size distributions in the material. Specifically, flaws with a size smaller than $c_{m c}$ [Fig. 5(b)] will be excluded from the multiple cracking process. As shown in Figs. 5(a and b), $c_{m c}$ is defined as the flaw size where the cracking stress level $\sigma_{f c}$ attains the maximum bridging stress $\sigma_{\text {peak }}$. Flaws smaller than $c_{m c}$ cannot be activated because they require an applied load higher than $\sigma_{\text {peak }}$ (Alwan 1994; Leung 1996). Therefore, knowing the probability description of the flaw size distribution and $c_{m c}$ leads to predicting unsaturated crack spacing.

By equating $\sigma_{f c}$ in (3) to $\sigma_{\text {peak }}$ in (6), $c_{m c}$ can be evaluated (Wu and Li 1995b)

$$
\left(\frac{2 \sqrt{2}}{3} \sqrt{\bar{c}_{m c}}-\frac{\bar{c}_{m c}}{4}\right)+\frac{\sqrt{\pi}}{2} \frac{\bar{K}}{\bar{c}_{m c}}=1
$$

The distribution of flaw size can be treated as a random process and expressed by the Weibull-type function (Weibull 1951)

$$
F(\bar{c})=\exp \left[-\frac{1}{\lambda}\left(\frac{\bar{c}_{0}}{\bar{c}}\right)^{m}\right]
$$

where $\lambda=$ scale factor; $m=$ Weibull modulus; and $\bar{c}_{0}=$ reference crack radius. The function $F$ in (11) represents the cumulative probability of flaws with normalized size less than $\bar{c}$ in the tensile specimen. This statistical distribution function, following that of Spearing and Zok (1993) and $\mathrm{Wu}$ and $\mathrm{Li}$ (1995b), is uniquely determined for a composite once the parameters $m, \lambda$, and $\bar{c}_{0}$ are specified. These three parameters may be expected to depend on composite characteristics such as mix proportion and mixing process. However, such dependency has not been investigated.

Once the flaw size distribution is identified, the theoretical unsaturated crack spacing $x_{d}^{\text {theory }}$ can finally be predicted for PSH-RSFRCCs in terms of saturated crack spacing $x_{d}$

$$
x_{d}^{\text {theory }}=\frac{x_{d}}{1-F\left(\bar{c}_{m c}\right)}
$$

Eq. (12) implies that $x_{d}^{\text {theory }}=x_{d}$ if all flaws are of size larger than $c_{m c}$, when $F=0$, corresponding to the fully saturated case. When some flaws are of a size less than $c_{m c}$, the case of unsaturated multiple cracking prevails. The function $F$ is schematically illustrated in Fig. 5(b), for three different flaw size distributions. This figure shows the effects of $\lambda$, the aforementioned scaling parameter of flaw size distribution function $F$ in (11). For higher values of $\lambda$, the flaw distribution is shifted toward a smaller size for given $m$ and $\bar{c}_{0}$, and $F\left(\bar{c}_{m c}\right)$ approaches unity. In this case $x_{d}^{\text {theory }}$ approaches infinity through (12). This result implies the single crack behavior of composites. On the other hand, low $\lambda$ leads to $F=0$, thus causing $x_{d}^{\text {theory }}=x_{d}$, which denotes saturated PSH behavior. However, moderate $\lambda$ leads to $0<F<1$, therefore $x_{d}^{\text {theory }}$ lies between $x_{d}$ and infinity.

As deduced above, the three parameters in (11) heavily affect $x_{d}^{\text {theory }}$; hence determining these three parameters is the last task to complete the current stress-strain theory. The detailed investigation for this determination is outside the scope of the present study. However, a phenomenological method is alternatively introduced, as described below.

\section{Identification of Flaw Size Distribution}

The current study proposes a simple procedure to identify the three parameters in the flaw size distribution function $F$ in (11) by modifying the approach in the literature ( $\mathrm{Wu}$ and $\mathrm{Li}$ $1995 b)$. Identifying $F$ needs to determine $m, \bar{c}_{0}$, and $\lambda$ as depicted in Step IV of Fig. 4. The parameter $m$ is identified essentially following the same approach as that in the literature
(Wu and $\mathrm{Li}$ 1995b). For concrete, $m$ is typically assumed to fall between 2 and 3, which is derived by converting magnitudes for a strength-based function (Ashby and Jones 1986). For RSFRCCs, $m=2$ is adopted as a slightly lower value considering additional flaws introduced due to fiber interference in the mixing process (Wu and Li 1995b). For the second parameter, the current study adopts $\bar{c}_{0}=\bar{c}_{m}$, where $\bar{c}_{m}$ corresponds to the critical flaw size responsible for tensile failure of matrix material (without fiber). The parameter $c_{m}$ can be predicted using basic linear elastic fracture mechanics

$$
c_{m}=\left(\frac{\sqrt{\pi}}{2} \frac{K_{m}}{\sigma_{m u}}\right)^{2}
$$

where $K_{m}=$ fracture toughness of matrix. This formula is derived by assuming a penny-shaped crack is involved in the infinite bulk solid of materials.

It should be noted that this study adopts $c_{0}=c_{m}$ instead of $c_{s}$, the crack size at steady-state cracking achieved in the literature (Spearing and Zok 1993; Wu and Li 1995b). This is because $c_{s}$ heavily depends on fiber bridging performance as well as matrix properties (i.e., composites having higher fiber bridging performance take lower $c_{s}$ ). The current study intends to eliminate this influence and to deal with $c_{0}$ as a matrixdependent parameter. The background of this modification is subsequently described in this section.

The last parameter $\lambda$ is handled as an adjusting parameter and is determined by the next procedure. It is inversely identified via (12) using the reference tensile test data from a sample PSH composite, for which crack spacing observation $x_{d}^{\text {test }}$ has been measured. The parameters $x_{d}$ and $\bar{c}_{m c}$ in (12) can be estimated using (9) and (10), respectively, when fundamental micromechanical parameters for fiber, fiber/matrix interface, and matrix are known. Therefore, setting $x_{d}^{\text {theory }}=x_{d}^{\text {test }}$ enables one to invert for $\lambda$.

The principle underlying idea in this approach is that the three parameters in (12) are supposed to be uniquely determined for similar composites. For example, having tensile test data from a sample PSH composite, one can first obtain the three flaw size distribution parameters for this sample composite by adopting the above procedure. Then $x_{d}^{\text {theory }}$ for similar composites may be estimated using these obtained parameters. In the current study, "similar" means having the same matrix mix proportion but being different in fiber volume fraction and/or fiber length. Indeed, flaw size distribution was demonstrated substantially depending on the matrix mix proportion and mix processing through previous research results of macrodefect free cement materials, in which the population of macroflaws was remarkably diminished by reducing the waterto-cement ratio and introducing a special mixing process (Kendall et al. 1983). However, it is still a concern that $\lambda$ cannot be uniquely determined even for similar composites. This concern arises because the fresh properties of composites are generally observed to be influenced by fiber length and fiber volume. This observation suggests that the flaw size distribution may also be influenced by fiber length and fiber volume. Nevertheless, the effect of these differences is subsequently demonstrated to be minor using analysis results via the next section.

It should be noted that $\bar{c}_{0}$ can be arbitrarily defined in the proposed procedure for identifying flaw size distribution $F$ when Weibull modulus $m$ is fixed. For a different definition of $\bar{c}_{0}=\bar{c}_{m}^{\prime}, \lambda^{\prime}$ can be determined as follows:

$$
\frac{\lambda^{\prime}}{\lambda}=\left(\frac{\bar{c}_{m}^{\prime}}{\bar{c}_{m}}\right)^{m}
$$

However, the three parameters contained in the function $F$ should eventually be expressed as a function of micromechanical parameters of composites after extending the proposed 
theory rather than using the phenomenological method. Therefore, as the most simple example of such an expression, this study adopted (13) with some physical meaning.

\section{THEORY VALIDATION}

\section{Scheme for Theory Validation}

The presented theory is substantiated by referring to two series of uniaxial tensile test results in which four different RSFRCC mixes involving high strength polyethylene fiber were employed. Details of these experiments are reported in the literature (Wu and Li 1995b; Kanda and Li 1998). In these tests, crack spacing has been recorded, and macroscopic stressstrain relations have been measured for all uniaxial tensile specimens. These tests were conducted at 4 weeks of age and involved similar composite constitutive properties, in which composites were processed in an Omni Mixer with a waterto-cement ratio of 0.27 . They are, however, different in fiber volume fraction $V_{f}$ (varying from 0.75 to $2.00 \%$ ) and fiber length $L_{f}(12.7$ to $19.1 \mathrm{~mm})$ as summarized in Table 1 . These specimens showed a broad range of multiple crack saturation intensity, and their test results are summarized in Table 2. These test results were obtained as the average of two specimens. It should be noted that the first test series in Table 1 by Kanda and Li (1998) originally involved two other composites with lower $V_{f}(0.5 \%)$ and younger curing age (1 week). However, these two composites were excluded in the discussion of this study. This is because the composite with $V_{f}=0.5 \%$ showed single cracking behavior without PSH, and the composite at 1 week of age was found to have a different flaw size distribution.

The current study utilizes the aforementioned experimental data in Table 2 for two purposes. First, the test result from a particular specimen $\left(V_{f}=2 \%\right.$ and $\left.L_{f}=12.7 \mathrm{~mm}\right)$ is chosen as the reference data to identify $\lambda$ and thus to determine flaw size distribution function $F$ of (11). This resulting $\lambda$ is referred to as $\lambda_{r e f}$. Second, the experimental data for the other similar composites were used to indicate the accuracy of the theoret-

TABLE 1. Summary of Composites Employed for Theory Validation

\begin{tabular}{c|c|c|l}
\hline \hline & $\begin{array}{c}\text { Fiber } \\
\text { volume } \\
\text { fraction } V_{f} \\
(\%)\end{array}$ & $\begin{array}{c}\text { Fiber } \\
\text { length } L_{f} \\
(\mathrm{~mm}) \\
(3)\end{array}$ & \multicolumn{1}{c}{$\begin{array}{c}\text { Reference } \\
(4)\end{array}$} \\
\hline $\begin{array}{c}\text { Composite } \\
(1)\end{array}$ & $(2)$ & 19.1 & Kanda and Li (1998) \\
$V_{f}=0.75 \%$ & 0.75 & 19.1 & Kanda and Li (1998) \\
$V_{f}=1.0 \%$ & 1.00 & 19.1 & Kanda and Li (1998) \\
$V_{f}=1.25 \%$ & 1.25 & 12.7 & Wu and Li (1995b) \\
$V_{f}=2.0 \%$ & 2.00 & \multicolumn{2}{|l}{} \\
\hline \hline
\end{tabular}

ical predictions. For composites other than the reference, $x_{d}^{\text {theory }}$ is estimated by substituting $\lambda_{\text {ref }}$ for $\lambda$ in (11) and then compared with the corresponding measured $x_{d}^{\text {test }}$. A ratio $x_{d}^{\text {theory }} / x_{d}^{\text {test }}$ close to unity indicates high fidelity of the simulation of crack spacing using the present theory.

\section{Prediction of Stress-Strain Relation}

The tensile test results for composites in Table 2 were analyzed following the aforementioned procedure. This analysis essentially employed micromechanical parameters specified in the sources (Wu and Li 1995b; Kanda and Li 1998). However, unspecified parameters were assumed by referring to other works in the literature. For example, the slip-hardening parameters $\beta_{1}$ and $\beta_{2}$ were determined by data-fitting of single fiber pullout test results, as shown in Fig. 8. These parameters are summarized in Table 3.

Theory validation is explained according to the procedure in Fig. 4. In the first step of Fig. 4, two major unknowns among the three, $\sigma_{s s}$ and $\sigma_{\text {peak }}$, were calculated and tabulated as in Table 4 . The remaining major unknown parameter, $\varepsilon_{c u}$, was obtained by reversing the steps from IV to I in Fig. 4, and its identification process is explained below.

First, the three parameters, $\lambda, c_{0}$, and $m$, were identified to determine the flaw size distribution function $F$ using the reference data (Step IV). In this step, $\lambda_{\text {ref }}=1.19$ was obtained via (12), where the reference data $\left(V_{f}=2 \%\right)$ indicated in Table 4 were used. Furthermore, the reference flaw size $c_{0}\left(=c_{m}\right)$ was calculated as $33 \mathrm{~mm}$ by means of (13). Then the assumption of $m=2$ led to the $F$-function shown in Fig. 9. This $F$-function indicates that approximately half of the flaw population, deduced from cumulative probability at $\bar{c} / \bar{c}_{0}=1$, is larger than $c_{m}$. The $F$-function thus obtained is assumed to be universal for all the specimens in this test series.

Second, the minimum flaw size $c_{m c}$ and saturated crack spacing $x_{d}$ were evaluated, and cumulative probability at $c_{m c}$

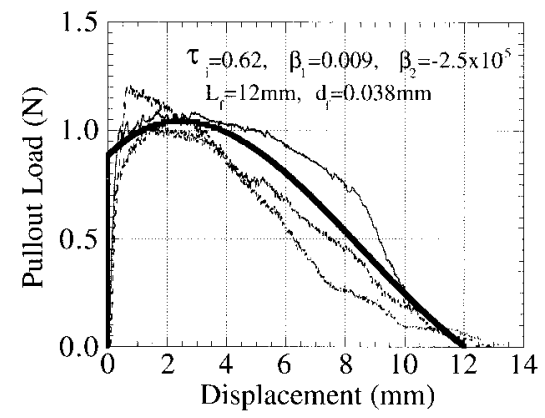

FIG. 8. Determination of Bond Properties by Fitting Single Fiber Pullout Test Data

TABLE 2. Summary of Composite Test Results

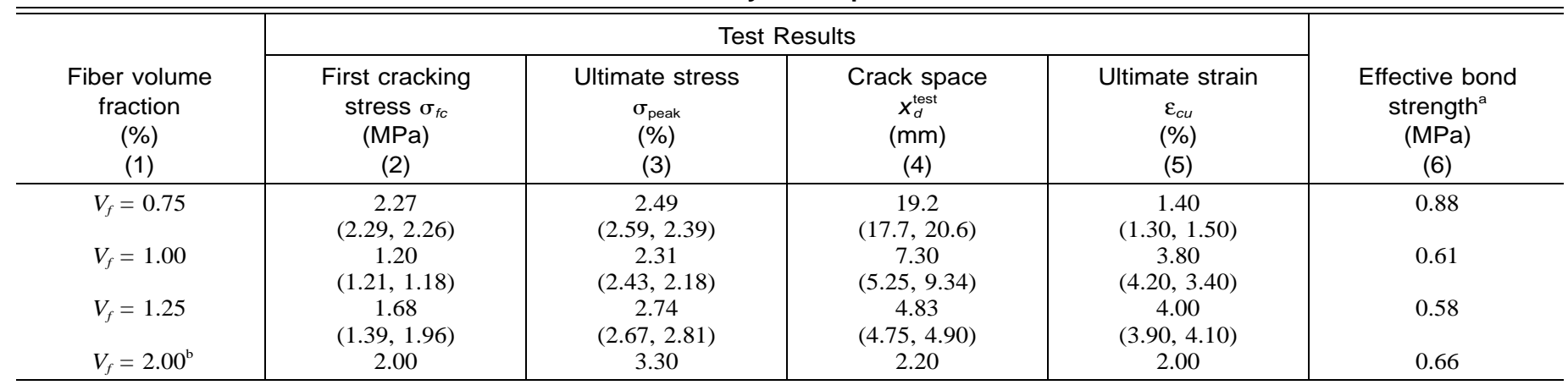

Note: Test results are average of two specimens, and data for individual specimens are in parentheses.

anverted by using Eq. (6).

${ }^{b}$ No individual data were shown in study of Wu and Li (1995b). 
TABLE 3. Micromechanical Parameters for Analysis

\begin{tabular}{l|l}
\hline \hline \multicolumn{1}{c|}{$\begin{array}{c}\text { Micromechanical parameter } \\
(1)\end{array}$} & $\begin{array}{c}\text { Polyethylene } \\
\text { composite } \\
(2)\end{array}$ \\
\hline Matrix elastic modulus $E_{m}(\mathrm{GPa})$ & 23 \\
Matrix fracture toughness $K_{m}\left(\mathrm{MPa} \mathrm{m} \mathrm{m}^{0.5}\right)$ & 0.33 \\
Matrix tensile strength $\sigma_{m u}(\mathrm{MPa})$ & 1.6 \\
Fiber elastic modulus $E_{f}(\mathrm{GPa})$ & 117 \\
Fiber volume fraction $V_{f}(\%)$ & $0.75-2.00$ \\
Fiber length $L_{f}(\mathrm{~mm})$ & $12.7-19.05$ \\
Fiber diameter $d_{f}(\mathrm{~mm})$ & 0.038 \\
Friction bond strength $\tau_{i}(\mathrm{MPa})$ & $0.62-0.66$ \\
Slip-hardening parameter $\beta_{1}$ & 0.009 \\
Slip-hardening parameter $\beta_{2}\left(\times 10^{-5}\right)$ & -2.5 \\
Snubbing coefficient $f$ & 0.5 \\
\hline \hline
\end{tabular}

[ $\left.=F\left(\bar{c}_{m c}\right)\right]$ was then evaluated by using the $F$-function obtained above (Step III). The parameter $c_{m c}$ was calculated by employing (10). Fig. 10 shows that $\bar{c}_{m c}$ generally decreases with fiber volume fraction $V_{f}$. This result implies that more flaws are to be activated in the multiple cracking process, and therefore greater intensity of multiple cracking can be expected for those composites with higher $V_{f}$. This expectation is consistent with the experimental observations (Kanda and Li 1998). Furthermore, the calculated $c_{m c}$ of a composite with $V_{f}=2 \%$ is larger than that with $V_{f}=1.25 \%$ (Fig. 10). This result is due to the shorter fiber length $L_{f}$ used for the composite with $V_{f}=$ $2 \%$. The potential for PSH behavior being more sensitive to $L_{f}$ than $V_{f}$ has been demonstrated in previous researches $(\mathrm{Li}$ and Leung 1992). Another unknown, $x_{d}$, was calculated using (9) and tabulated in Table 4. It is shown that $x_{d}$ decreases with increasing $V_{f}$. In Fig. 9, $\bar{c}_{m c} / \bar{c}_{0}=1.38$ and 0.745 are indicated with vertical arrows for $V_{f}=0.75$ and $1.25 \%$, respectively. The corresponding calculated values of $F\left(\bar{c}_{m c}\right)$ are 0.646 and 0.219 for these composites. Lower probability was calculated for $V_{f}$ $=1.25 \%$ than for $V_{f}=0.75 \%$. This means that more flaws, which are eventually activated in the multiple cracking process, have size beyond $c_{m c}$ for $V_{f}=1.25 \%$, than for $V_{f}=0.75 \%$. The calculation of this result agrees with the observation in the experiment in which a larger number of cracks was recorded for $V_{f}=1.25 \%$ (Kanda and Li 1998). For other composites, $F\left(\bar{c}_{m c}\right)$ was calculated and tabulated in Table 4. Notice that $F\left(\bar{c}_{m c}\right)$ for $V_{f}=2 \%$ is identical to $\left(1-x_{d}^{\text {theory }} / x_{d}\right)$ as the data of this composite were used to determine the $\lambda_{r e f}$ in the $F$-function.

Third, for Step II in Fig. 4, the ultimate COD $\delta_{\text {peak }}$ calculated is summarized in Table 4 . Then $x_{d}^{\text {theory }}$ was evaluated as illustrated in Fig. 11, in which $x_{d}^{\text {theory }}$ is compared with the experimentally observed crack spacing $x_{d}^{\text {test }}$. The theoretical prediction appears to be consistent with the test results.

Finally, going back to Step I in Fig. 4, $\varepsilon_{c u}$ was calculated using the $x_{d}$ just computed in (7). The theoretical results of $\varepsilon_{c u}$ is illustrated in Fig. 12. As shown in this figure, the theoretical prediction of crack spacing is generally consistent with that from the test results. Therefore, the three major unknowns, $\sigma_{s s}$, $\sigma_{\text {peak }}$, and $\varepsilon_{c u}$, were theoretically evaluated, thus enabling one

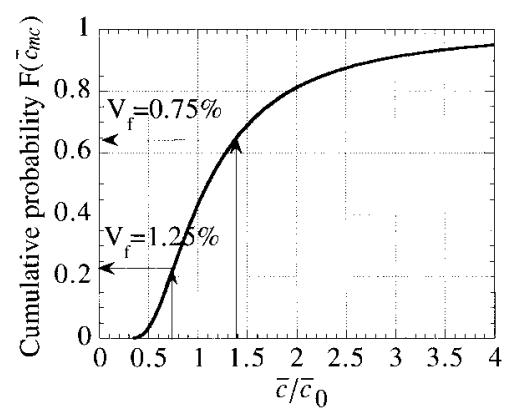

FIG. 9. Determined Flaw Size Distribution and $\boldsymbol{F}\left(\bar{c}_{m c}\right)$

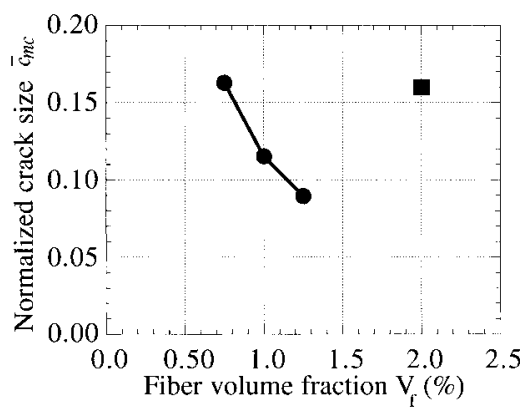

FIG. 10. Analyzed Results for Minimum Activated Flaw Size; $\bullet: L_{f}=19.05 \mathrm{~mm}$; $: L_{f}=\mathbf{1 2 . 7} \mathrm{mm}$

to predict the stress-strain relation of PSH-RSFRCCs using (1).

The predicted stress-strain relations were compared with the experimentally measured curves in Figs. 13(a-c), for the composites with $V_{f}=0.75,1.0$, and $1.25 \%$, respectively. These figures indicate that the theoretical prediction captures the trend of the test results for the three cases. However, stress performance for the $V_{f}=0.75 \%$ [Fig. 13(a)] and the strain capacity for the $V_{f}=1.25 \%$ specimen [Fig. 13(c)] were not necessarily predicted with sufficient accuracy. The stress-strain relation was not estimated for $V_{f}=2 \%$ as these data were used to identify the $F$-function.

One reason for these prediction errors may be in the method of determining fiber/matrix interfacial properties such as bond strength $\tau_{i}$, slip-hardening parameters $\beta_{1}$ and $\beta_{2}$, which employed single fiber pullout test (Katz and Li 1996). The interface properties indicated in Table 3 neglect deterioration by fiber-fiber interaction in the actual composites (Kanda and $\mathrm{Li}$ 1998). For example, effective bond strengths for $V_{f}=0.75 \%$, which were inverted from (6) by substituting test data to $\sigma_{\text {peak }}$, were found to be much higher than assumed $\tau_{i}$ as shown in Table 2.

Note that the obtained $c_{m}$ with (13) is too large compared with the specimen size, which is $76 \times 36 \mathrm{~mm}$ in cross section. This discrepancy appears to be attributed to the hypothesis adopted in (13), in which a penny-shaped single crack is assumed to be involved in the infinite bulk solid of materials for simplicity. In reality, however, numerous cracks are present; their interaction increases the stress intensity factor. Specifi-

TABLE 4. Analysis Results

\begin{tabular}{|c|c|c|c|c|c|c|c|}
\hline $\begin{array}{c}\text { Composite } \\
\text { (1) }\end{array}$ & $\begin{array}{c}\text { Steady-state } \\
\text { cracking stress } \\
\sigma_{s s} \\
(\mathrm{MPa}) \\
(2)\end{array}$ & $\begin{array}{l}\text { Ultimate stress } \\
\qquad \begin{array}{c}\sigma_{\text {peak }} \\
(\mathrm{MPa}) \\
(3)\end{array}\end{array}$ & $\begin{array}{c}\text { Minimum flaw } \\
\text { size activate } \\
\bar{c}_{m c} \\
(4)\end{array}$ & $\begin{array}{c}\text { Saturated ultimate } \\
\text { crack space } \\
x_{d} \\
(\mathrm{~mm}) \\
(5)\end{array}$ & $\begin{array}{l}\text { Ultimate COD } \\
\qquad \begin{array}{c}\delta_{\text {peak }} \\
(\mu \mathrm{m}) \\
(6)\end{array}\end{array}$ & $\begin{array}{c}F\left(\bar{c}_{m c}\right) \\
(7)\end{array}$ & $\begin{array}{c}\text { Ultimate } \\
\text { crack space } \\
x_{d}^{\text {theory }} \\
(\mathrm{mm}) \\
(8)\end{array}$ \\
\hline $\begin{aligned} V_{f} & =0.75 \% \\
V_{f} & =1.0 \% \\
V_{f} & =1.25 \% \\
V_{f} & =2.0 \%^{\mathrm{a}}\end{aligned}$ & $\begin{array}{l}1.33 \\
1.64 \\
1.93 \\
2.50\end{array}$ & $\begin{array}{l}1.75 \\
2.34 \\
2.92 \\
3.31\end{array}$ & $\begin{array}{l}0.163 \\
0.115 \\
0.089 \\
0.160\end{array}$ & $\begin{array}{l}6.61 \\
4.12 \\
3.07 \\
1.74\end{array}$ & $\begin{array}{l}245 \\
2.45 \\
2.45 \\
22.5\end{array}$ & $\begin{array}{c}0.646 \\
0.410 \\
0.219 \\
-\end{array}$ & $\begin{array}{l}18.7 \\
6.98 \\
3.93 \\
-\end{array}$ \\
\hline
\end{tabular}

${ }^{\text {a }}$ Selected as reference composite and no calculation was made for $F\left(\bar{c}_{m c}\right)$ and $x_{d}^{\text {theory }}$. 


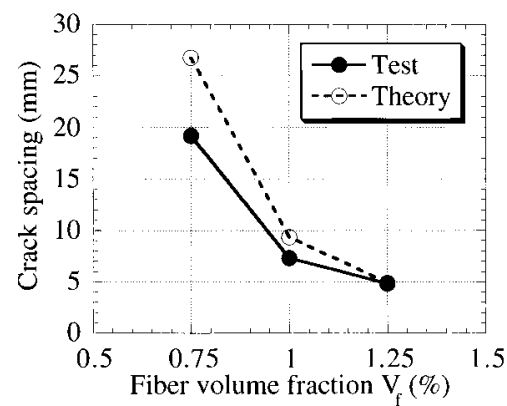

FIG. 11. Crack Spacing Theoretical and Experimental Results

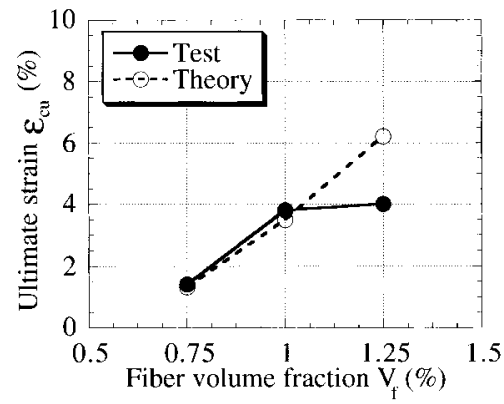

FIG. 12. Predicted Ultimate Strain Based on $\boldsymbol{\lambda}_{\text {ref }}$
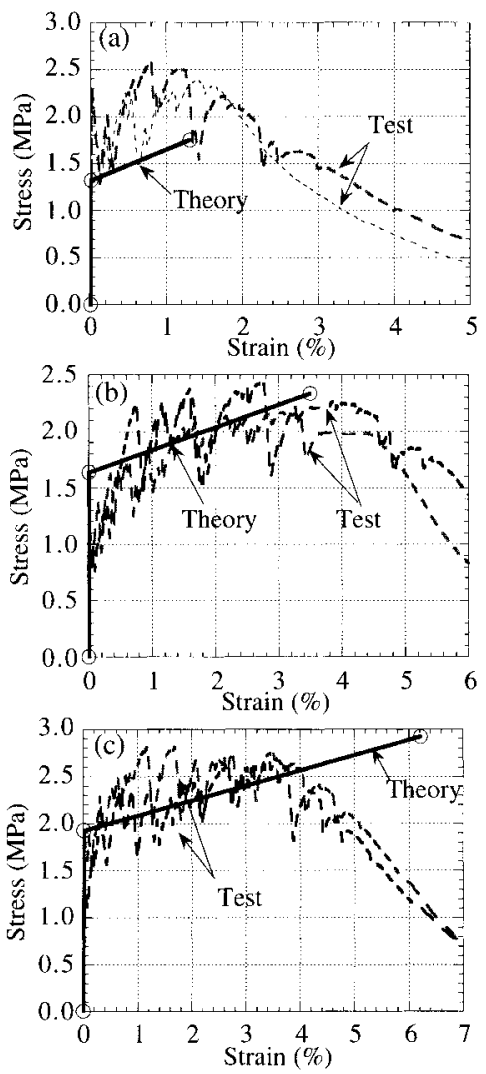

FIG. 13. Stress-Strain Relation Comparison between Test and Theory: (a) $V_{f}=0.75 \%$; (b) $V_{f}=1.00 \%$; (c) $V_{f}=1.25 \%$

cally, using linear elastic fracture mechanics, the actual flaw size $a$ (actual) $=a / R^{2}$, where $R$ is the ratio of the correction coefficient in the stress intensity factor expressions between the case of interaction cracks to the case of an isolated crack. For $R=1,3,5$, and 10, the corresponding $a$ (actual) will be $33,3.7,1.3$, and $0.33 \mathrm{~mm}$. The value of $R$ depends on the spacing between adjacent interacting cracks. Therefore, the actual flaw size responsible for matrix failure is considered much smaller than that obtained with (13). Nevertheless, it is im-
TABLE 5. Parameter $\lambda$ Based on Multiple Crack Spacing Data Set from Different Composites

\begin{tabular}{c|c}
\hline \hline Reference & \multicolumn{1}{|c}{$\lambda$} \\
$(1)$ & $(2)$ \\
\hline$V_{f}=0.75 \%$ & 1.23 \\
$V_{f}=1.0 \%$ & 1.28 \\
$V_{f}=1.25 \%$ & 1.79 \\
$V_{f}=2.0 \%$ & $1.192\left(\lambda_{r e f}\right)$ \\
\hline \hline
\end{tabular}

portant that the minimum activating flaw size $c_{m c}$ is estimated with (10) under the same hypothesis as (13) ( $\mathrm{Li}$ and Leung 1992). Hence, $c_{m}$ and $c_{m c}$ are considered equally overestimated, and the relationship between these flaw sizes demonstrated in Fig. 9 appears to reflect that in the actual composites. However, a more advanced hypothesis (e.g., random flaws interacting with each other in composites) is needed to more accurately determine $c_{m c}$ and flaw size distribution function $F$ in order to improve modeling precision of the stress-strain relation of PSH-RSFRCCs.

\section{Identifying Flaw Size Distribution and Remaining Problems}

The adopted hypothesis of a unique $F$-function describing the flaw size distribution for similar composites should be checked. For this verification, $\lambda$ was first calculated for the various specimens following the same procedure as described in the previous subsection. The calculated values of $\lambda$ are summarized in Table 5, showing that the results of $\lambda$ are very similar among all the composites. Indeed, the ultimate strain capacity $\varepsilon_{c u}^{\text {theory }}$, predicted using different values of $\lambda$ in Table 5 , was reasonably consistent with test results similar to Fig. 12 . Hence, it may be concluded that $\varepsilon_{c u}^{\text {theory }}$ was reproducible regardless of reference data selections with similar composites.

The limited data reveal that the presented theory reasonably predicts the tensile stress-strain relation of PSH-RSFRCCs having a similar configuration (matrix mix proportion, mixing process, and age) but is different in fiber volume fraction and/ or fiber length. However, the accuracy of this estimation is not completely satisfactory. The discrepancies with the experimental data may have been contributed by the individual errors at different steps in the estimation process shown in Fig. 4, as well as those in interface property identification described in Fig. 13. Therefore, accuracy needs to be improved in each of Steps I-IV. This improvement is left for subsequent studies.

Furthermore, the tensile properties of PSH-RSFRCCs tend to have rather large variability as implied by Table 2 . The reliability of PSH-RSFRCC's tensile properties have not been fully understood as PSH-RSFRCC is a new material with a history of $<15$ years. This statistical evaluation should be achieved based on the clear mechanism understanding such as indicated in this paper for a next stage. In the next stage, the variability of micromechanical parameters, other than flaw size, should be clarified and taken into account in theory.

\section{DESIGN IMPLICATIONS}

The proposed theory facilitates the design of PSHRSFRCCs to achieve the required material properties that might be desirable in a given structure. For example, Maalej and $\mathrm{Li}$ (1995) studied a reinforced concrete beam designed to limit the COD in the concrete cover (replaced by PSHRSFRCC) when subjected to flexural load. The COD restriction was aimed at increasing durability of this beam element under the attack of harmful chemical substances by limiting diffusion of those substances into the element. The design ensures that the COD of the PSH-RSFRCC cover would not reach the ultimate value $\delta_{\text {peak }}$ even at the structural ultimate 


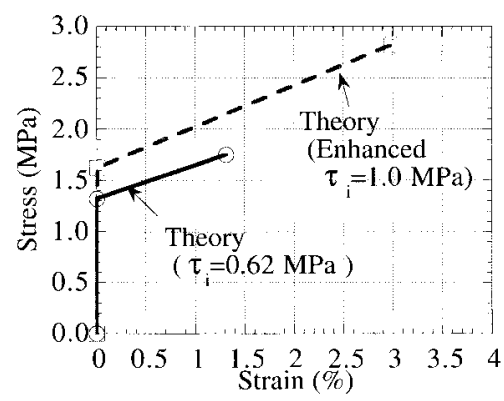

FIG. 14. Influence of Interface Bond on Resulting StressStrain Relation (Theory)

state of the beam. Furthermore, it is necessary to tailor the composite such that $\varepsilon_{c u}$ exceeds the maximum strain expected in the cover of the beam, so that strain localization would not occur in the PSH-RSFRCC. By linking $\delta_{\text {peak }}$ and $\varepsilon_{c u}$ to micromechanical properties, it is possible to tailor the cementitious composite to meet the intended structural durability requirement of the reinforced concrete beam.

Controlling $\varepsilon_{c u}$ can be achieved through modifying either $x_{d}^{\text {theory }}$ or $\delta_{\text {peak }}$, which are expressed as functions of micromechanical parameters in the proposed theory. This modification is demonstrated in Fig. 14, which includes the theoretically predicted stress-strain relations of the composite with $V_{f}=$ $0.75 \%$. For this composite, $\varepsilon_{c u}$ is shown to be predicted around $1 \%$ in Fig. 14, and this may not be sufficient for certain applications. In such a case, $\varepsilon_{c u}$ should be extended, which may be achieved in various ways. The most common approach is to increase $V_{f}$, whose effect is demonstrated in Fig. 12. However, this approach does not always work (e.g., due to the deterioration of workability with increasing fiber content or due to the higher cost of fibers). Under this restriction, the proposed theory facilitates tailoring composite constituents such as frictional bond strength $\tau_{i}$, which can be enhanced to a few times larger by using techniques such as plasma treatment of fibers (Li et al. 1996) to effectively increase $\varepsilon_{c u}$. The effect of increasing $\tau_{i}$ is rather remarkable as demonstrated in Fig. 14, which shows that $\varepsilon_{c u}$ is improved from about 1.5 to $3 \%$ when $\tau_{i}$ is enhanced from 0.62 to $1.0 \mathrm{MPa}$. Hence, the proposed theory can be a powerful tool to effectively tailor composite property for targeted structural performance.

\section{CONCLUSIONS}

This study proposes a new comprehensive approach to predicting the stress-strain relation of PSH-RSFRCCs, in which the stress-strain relation is assumed to be a bilinear line. This approach requires theoretical modeling of the ultimate crack spacing $x_{d}^{\text {theory }}$. The model employs a probabilistic description, in which the Weibull function is adopted to represent flaw size distribution. Identifying the parameters in this function requires at least one set of experimental data on crack spacing. Subsequently, the crack spacing and stress-strain curves can be predicted for similar composites with different fiber content and/or length. The validity of the proposed approach has been substantiated with the experimental results. This study reveals the following:

- Flaw size distribution can be assumed unique for similar composites (with identical matrix mix proportion, mixing process, and age) even when different in fiber length and fiber volume fraction.

- Ultimate crack spacing is found to be consistent with the test results by means of the proposed crack spacing theory.

- The experimental stress-strain relation of PSH-RSFRCCs is found to be reproducible by employing the proposed theoretical approach with reasonable accuracy.

- The proposed approach for the stress-strain relation has been indicated as a potentially powerful tool for tailoring composites in order to satisfy targeted structural performance.

The proposed stress-strain model contributes to a better understanding of PSH-RSFRCC's tensile behavior and should extend the applications of these composites. However, the accuracy in predicting the stress-strain relation can be further improved by taking into account factors that are neglected in the current study, such as the variability of micromechanics parameters other than flaw size. This refinement is left for subsequent studies.

\section{ACKNOWLEDGMENTS}

This work has been partially supported by a grant (NSF-G-CMS9601262) from the National Science Foundation to the University of Michigan, Ann Arbor, Mich. This work was completed while V. Li was a visiting professor at the University of Tokyo, Japan.

\section{APPENDIX I. REFERENCES}

Alwan, J. M. (1994). "Modeling of the mechanical behavior of fiber reinforced cement based composites under tensile loads," $\mathrm{PhD}$ thesis, Dept. of Civ. and Envir. Engrg., University of Michigan, Ann Arbor, Mich.

Ashby, M. F., and Jones, D. R. H. (1986). Engineering materials, 2, Pergamon, Tarrytown, N.Y.

Aveston, J., Cooper, G. A., and Kelly, A. (1971). "Single and multiple fracture." The properties of fiber composites, IPC Science and Technology Press, Guildford, U.K., 15-26.

Aveston, J., and Kelly, A. (1973). "Theory for multiple fracture of fibrous composites." J. Mat. Sci., 8, 352-362.

Beyerle, D. S., Spearing, S. M., Zok, F. W., and Evans, A. G. (1992). "Damage and failure in unidirectional ceramic-matrix composites." $J$. Am. Ceramic Soc., 75(10), 2719-2725.

Cho, C., Holmes, J. W., and Barber, J. R. (1992). "Distribution of matrix cracks in a uniaxial ceramic composite." J. Am. Ceramic Soc., 75(2), 316-324.

Evans, A. G., Domergue, J. M., and Vagaggini, E. (1994). "Methodology for relating the tensile constitutive behavior of ceramic matrix composites to constituent properties." J. Am. Ceramic Soc., 77(6), 14251435.

He, M. Y., Wu, B. X., Evans, A. G., and Hutchinson, J. W. (1994). "Inelastic strains due to matrix cracking in unidirectional fiber-reinforced composites." Mech. of Mat., 18, 213-229.

Kabele, P., and Horii, H. (1996). "Analytical model for fracture behaviors of pseudo strain-hardening cementitious composites." J. Mat., Conc. Struct., Pavements, Tokyo, 30(532), 209-219.

Kanda, T., and Li, V. C. (1998). "Multiple cracking sequence and saturation in fiber reinforced cementitious composites." Concrete Res. and Technol., Tokyo, 9(2), 1-15.

Katz, A., and Li, V. C. (1996). "A special technique for determining the bond strength of carbon fibers in cement matrix by pullout test." $J$. Mat. Sci. Letters, 15, 1821-1823.

Kendall, K., Howard, A. J., and Birchall, J. D. (1983). "The relation between porosity, microstructure and strength, and the approach to advanced cement-based materials." Philosophical Trans. Royal Soc., London, A310, 139-153.

Kullaa, J. (1998). "Micromechanics of multiple cracking-Part II: Statistical tensile behavior." J. Mat. Sci., 33, 4225-4234.

Leung, C. K. Y. (1996). "Design criteria for pseudoductile fiber-reinforced composites." J. Engrg. Mech., ASCE, 122(1), 10-18.

Li, V. C. (1993). "From micromechanics to structural engineering-The design of cementitious composites for civil engineering applications." J. Struct. Mech. and Earthquake Engrg., Tokyo, 10(2), 37-48.

Li, V. C., and Leung, C. K. Y. (1992). "Steady-state and multiple cracking of short random fiber composites." J. Engrg. Mech., ASCE, 118(11), 2246-2264.

Li, V. C., Mishra, D. K., and Wu, H. C. (1995). "Matrix design for pseudo-strain hardening fiber reinforced cementitious composites." Mat. and Struct., Paris, 28(183), 586-595.

Li, V. C., and Stang, H. (1996). "Interface property characterization and strengthening mechanisms in fiber reinforced cement based composites." J. Advanced Cement Based Mat., 6(2), 1-20. 
Li, V. C., Wu, H. C., and Chan, Y. W. (1996). "Effect of plasma treatment of polyethylene fibers on interface and cementitious composite properties." J. Am. Ceramics Soc., 79(3), 700-704.

Lin, Z., and Li, V. C. (1997). "Crack bridging in fiber reinforced cementitious composites with slip-hardening interfaces." J. Mech. Phys. of Solids, 45(5), 763-787.

Maalej, M., and Li, V. C. (1995). "Introduction of strain hardening engineered cementitious composites in the design of reinforced concrete flexural members for improved durability." Am. Concrete Inst. Struct. J., 92(2), 167-176.

Marshall, D. B., and Cox, B. N. (1987). "Tensile fracture of brittle matrix composites: Influence of fiber strength." Acta Metallurgica, 35(11), 2607-2619.

Marshall, D. B., Cox, B. N., and Evans, A. G. (1985). "The mechanics of matrix cracking in brittle-matrix fiber composites." Acta Metallurgica, 33(11), 2013-2021.

Naaman, A. E., and Najm, H. (1991). "Bond-slip mechanisms of steel fibers in concrete." ACI Mat. J., 88(2), 135-145.

Naaman, A. E., and Reinhardt, H. W. (1995). "High performance fiber reinforced cement composites 2 (HPFRCC2)." RILEM Proc., 31, E \& FN Spon, London, 291-348.

Spearing, S. M., and Zok, F. W. (1993). "Stochastic aspects of matrix cracking in brittle matrix composites." J. Engrg. Mat. and Technol., 115, 314-318.

Weibull, W. (1951). "A statistical distribution function of wide applicability." J. Appl. Mech., 18, 293.

Wu, H.C., and Li, V. C. (1995a). "Snubbing and bundling effects on multiple crack spacing of discontinuous random fiber-reinforced brittle matrix composites." J. Am. Ceramic Soc., 75(12), 34873489.

Wu, H. C., and Li, V. C. (1995b). "Stochastic process of multiple cracking in discontinuous random fiber reinforced brittle matrix composites." Int. J. Damage Mech., 4(1), 83-102.

Zok, F. W., and Spearing, S. M. (1992). "Matrix crack spacing in brittle matrix composites." Acta Metallurgica et Materialia, 40(8), 20332043.

\section{APPENDIX II. NOTATION}

The following symbols are used in this paper:

$c=$ flaw radius;

$c_{m}=$ flaw radius responsible for matrix cracking;

$c_{m c}=$ minimum radius of flaw activated during multiple cracking process;

$c_{s}=$ flaw radius when steady-state cracking occurs;

$c_{0}=$ reference flaw radius;

$d_{f}=$ fiber diameter;

$E_{c}=$ elastic modulus of composite;

$E_{f}=$ elastic modulus of fiber;

$\mathbf{E}_{m}=$ elastic modulus of matrix;

$\mathbf{K}_{m}=$ matrix fracture toughness;

$K_{\text {tip }}=$ crack tip fracture toughness;

$L_{f}=$ fiber length;

$m=$ Weibull modulus;

$V_{f}=$ volume fraction of fiber;

$\mathbf{V}_{m}=$ volume fraction of matrix;

$x_{d}=$ saturated ultimate crack spacing;

$x_{d}^{\text {test }}=$ ultimate crack spacing observed in test;

$x_{d}^{\text {theory }}=$ theoretical prediction of ultimate crack spacing;

$\delta=$ crack opening displacement;

$\delta_{\text {peak }}=$ crack opening displacement at $\sigma_{\text {peak }}$;

$\varepsilon=$ strain of composite;

$\varepsilon_{c u}=$ ultimate strain of composite;

$\lambda=$ scale factor for flaw size distribution function;

$v=$ Poisson's ratio of composite;

$\sigma_{c}=$ crack bridging stress of composite;

$\sigma_{f c}=$ cracking stress level of composite;

$\boldsymbol{\sigma}_{m u}=$ matrix tensile strength;

$\sigma_{\text {peak }}=$ peak bridging stress of composite;

$\sigma_{s s}=$ steady-state cracking stress of composite; and

$\tau_{i}=$ frictional bond strength. 